\title{
Search for emission-line galaxies towards nearby voids. List $2^{\star}$
}

\author{
C.C. Popescu ${ }^{1,5}$, U. Hopp ${ }^{2}$, H.J. Hagen ${ }^{3}$, and H. Elsässer ${ }^{4}$ \\ 1 Max Planck Institut für Kernphysik, Saupfercheckweg 1, D-69117 Heidelberg, Germany \\ 2 Universitätssternwarte München, Scheiner Str.1, D-81679 München, Germany \\ 3 Hamburger Sternwarte, Gojenbergsweg 112, D-21029 Hamburg, Germany \\ 4 Max-Planck Institut für Astronomie, Königstuhl 17, D-69117 Heidelberg, Germany \\ 5 The Astronomical Institute of the Romanian Academy, Str. Cuţitul de Argint 5, 75212 Bucharest, Romania
}

Received April 3; accepted May 12, 1998

\begin{abstract}
We present a second list of emission-line galaxies (ELGs) discovered during our survey of nearby voids. The project aims to answer the question of whether the giant galaxies are fair tracers of the large scale structure and whether the voids are really empty. This paper is the third of a series of papers dedicated to this project. The survey was based on a sample selected from the HQS (Hamburg Quasar Survey) - IIIa-J digitised objective prism plates and the main selection criteria was the presence of emission-lines, mainly of the [OIII] $\lambda 5007$ line.

Positions, heliocentric redshifts, detected emissionlines and finding charts are provided for ELGs in a region North of the Coma Supercluster, $30.5^{\circ}<\delta<45.5^{\circ}$, centred around $13.5^{\mathrm{h}}$. Most of the galaxies presented here are from the extreme faint candidates, close to the detection limit of the photographic plates. Brighter galaxies detected among the candidates of the same region were discussed in Popescu et al. (1996) (Paper I). A few redshifts of other emission line galaxies observed since the publication of Paper I are also included; in total, we present new redshifts for a total of 47 galaxies.
\end{abstract}

Key words: large scale structure — galaxies: redshift surveys - galaxies: irregular

\section{Introduction}

Our search for emission-line galaxies (ELGs) towards nearby voids has been described in detail by Popescu et al. (1996) (Paper I) and Popescu et al. (1997) (Paper II).

Send offprint requests to: C.C. Popescu (e-mail: popescu@levi.mpi-hd.mpg.de)

* Based on observations obtained at the German-Spanish Observatory at Calar Alto, Almeria, Spain.
The project belongs to a larger one that has the aim of finding faint galaxies within the voids (Hopp et al. 1995; Kuhn et al. 1997). In summary, we have undertaken a program to search for dwarf galaxies in voids. The motivation of the project was to examine the question of whether the void regions represent real structures, or whether they merely appear as such due to selection effects. We have especially selected nearby voids which were very well defined in the distribution of normal giant galaxies, in order to overcome some of the limitations of previous surveys. Since there was a hint that emission-line galaxies tend to be less clustered, we chose to search for emission-line objects, but with the aim of finding mainly HII galaxies or BCDs (blue compact dwarf galaxies).

The candidates were selected from the objective prism plates taken from the Hamburg Quasar Survey (HQS) (Hagen et al. 1995). An automated procedure was applied to the low-resolution digitised objective-prism spectra, based on two parameters, the slope of the continuum and the "intensity" of the integrated spectra (see Paper I). The selected candidates were afterwards rescanned with high resolution and the final selected spectra were visually inspected for the presence of emission-lines. The candidates are mainly selected on the presence of the [OIII] $\lambda 5007$ line. In Paper I we presented the catalogue of all emission-line galaxies found with the above mentioned selection criteria and we will refer to this as "List 1". This means that objects fainter than our threshold in "intensity" were not considered. The cut in brightness produces some loss of very faint ELGs, having very little continuum and almost all the flux in the emission-lines. In order to prevent the latter incompleteness we also scanned the photographic plates for such faint objects. We observed all the new candidates with follow-up spectroscopy and produced a second list of ELGs that is given in the present paper. We will refer to this catalogue as "List 2". The extra survey was done only for one of our regions - Region 3 from Paper I (a region North of the Coma Supercluster, 
$30.5^{\circ}<\delta<45.5^{\circ}$, centred around $\left.13.5^{\mathrm{h}}\right)$. The results concerning the spatial distribution of all the galaxies found in our Region 3 ("List 1" and "List 2") were presented in Paper II. From the estimates of the expected number of void galaxies we concluded that we did not find an underlying homogeneous void population.

\section{Follow-up spectroscopy}

We observed all the candidates from the "List 2" during one observing run, between 20-24 May 1996. The observations were carried out with the CAFOS (The Calar Alto Faint Object Spectrograph) at the $2.2 \mathrm{~m}$ telescope at the German-Spanish Observatory at Calar Alto (Almeria, Spain). The details of the observations are given in Table 1. The observations from May 1996 were done in excellent weather conditions (the photometry being better than 5\%) and therefore the campaign was not dedicated to the follow-up spectroscopy of the new candidates but mainly to the spectrophotometry of a bigger sample of emission-line objects, including objects from Paper I. Because the main goal was accurate flux determination, we used a $4^{\prime \prime}$ slit widths, which on the the other hand increased the error in the redshift determination of the new candidates. Thus the error in redshift of the objects presented in this paper is bigger than the error of the objects from Paper I.

Table 1. The details of the spectroscopic observations from May 1996

\begin{tabular}{ll}
\hline \hline & \\
Detector & Lor-80 \\
Pixel Size $(\mu)$ & 15 \\
Slit width $(\prime \prime)$ & 4 \\
PA & 90. \\
Pixel number & $1024 \times 1024$ \\
Grism & 9 \\
Dispersion $(\AA /$ pixel $)$ & 5.6 \\
Resolution $(\AA)$ & 17 \\
Spectral Range $(\AA)$ & $3600-9000$ \\
& \\
\hline
\end{tabular}

The data were reduced using the MIDAS routines. The frames were biased and flatfield-corrected. For the extraction of the 1-dimensional spectra from the 2-dimensional data, the optimal extraction algorithm of Horne (1986) was used. The spectra were rebinned to a linear wavelength scale using a third or fifth order polynomial fitted to the dispersion curve of the comparison spectra. A flux calibration was applied, including the correction for the atmospheric extinction using the mean extinction values for Calar Alto. Finally the wavelength scale was checked by comparison with the night-sky lines. A more detailed description of the reduction procedure is given by Stickel et al. (1993).

Once fully reduced, the emission lines in each spectrum were measured by fitting a Gaussian Curve. The quoted redshifts were derived as means of the redshifts determined from the individual strong lines, and the errors of the redshifts were calculated as error of the mean. The observed redshifts were further corrected for the motion of the Earth and transformed in heliocentric redshifts. The internal errors from the May 1996 campaign are around $\Delta z=0.0002$. In order to estimate the external errors due to the $4^{\prime \prime}$ slit width we compared the redshifts of some galaxies observed also in a previous campaign (see Paper I) with a 2 " slit width. We obtained a total error of $\Delta z=0.00035$. For the few galaxies with absorption lines the errors are larger, up to $\Delta z=0.0007$.

\section{The catalogue}

The observed objects are listed in Table 2. The table is organised as follow:

- Column (1) gives the name of our objects, which are designated by the prefix HS (from Hamburg Survey), followed by the first four digits of the 1950.0 right ascension and declination.

- Column (2) gives alternative designations for those galaxies contained in other catalogues. The abbreviations are explained in the List of Abbreviations.

- Columns (3) and (4) give the 1950.0 positions. The coordinates are derived from the Hamburg direct plates and have an accuracy of $\pm 2^{\prime \prime}$.

- Column (5) gives the heliocentric redshifts.

- Column (6) contains a flag S (from selection) that gives the selection criteria used to select the candidates from the objective prism spectra: $\mathrm{E}$ for emission candidate (first priority) and B for blue candidate (second priority) (see Paper I).

- Column (7) contains special remarks.

Table 2 contains 39 galaxies, of which 33 are ELGs and 6 are galaxies with absorption lines. The later came only accidentally into our sample, as failures of our selection procedure. Three objects were previously known as IRAS sources and of these, two were also radio sources. From the 39 galaxies from our Table 2 only three were already known in the literature with available redshifts. These objects were therefore not reobserved, and the redshift given in the table is taken from literature.

One object from our catalogue, HS1337+3941 happens to be a very nearby dwarf galaxy. With a redshift of $z=0.0025$, this galaxy entered into our sample only as a second priority object (see Paper I for the selection flag $\mathrm{S}=\mathrm{E}$ ). It has a very faint [OIII] 5007 line and would be better detected by an $\mathrm{H} \alpha$ survey.

Finding charts of all our newly discovered objects can be found in Fig. 1. We also give the finding charts of 
Table 6. The details of the spectroscopic observations from Aug. 1995

\begin{tabular}{|c|c|c|}
\hline Detector & TEK & TEK \\
\hline Pixel Size $(\mu)$ & 24 & 24 \\
\hline Slit width (II) & 2.4 & 3.6 \\
\hline $\mathrm{PA}$ & various & various \\
\hline Pixel number & $1024 \times 1024$ & $512 \times 512$ \\
\hline Grating & $\# 10$ & $\# 5$ \\
\hline Dispersion $(\AA /$ pixel $)$ & 0.64 & 0.89 \\
\hline Resolution $(\AA)$ & 1.3 & 2.5 \\
\hline Spectral Range $(\AA)$ & $4820-5471$ & $4762-5672$ \\
\hline
\end{tabular}

Table 8. Candidates with available redshifts from literature, observed with the purpose of testing the redshift accuracy

\begin{tabular}{lllll}
\hline \hline name & $\begin{array}{l}\text { other } \\
\text { name }\end{array}$ & $\begin{array}{l}\text { our } \\
z\end{array}$ & $\begin{array}{l}\text { reference } \\
z\end{array}$ & $\begin{array}{l}\text { difference } \\
\text { in } z\end{array}$ \\
\hline \hline & & & & \\
HS0019+0450 & UM 27 & 0.0345 & 0.0340 & 0.0005 \\
HS0022+0014 & - & 0.0139 & 0.0139 & 0.0000 \\
HS0025+0443 & UM 40 & 0.0044 & 0.0045 & 0.0001 \\
HS0832+6624 & MRK 93 & 0.0176 & 0.0176 & 0.0000 \\
& & & & \\
\hline
\end{tabular}

some of our objects that were previously known as IRAS sources. The finding charts were prepared by means of the Palomar Sky Survey plates, digitised and distributed on CD-ROM by the Space Telescope Science Institute. Fields are $10^{\prime} \times 10^{\prime}$. North is up, and east is to the left.

The identified emission (absorption) lines of all the newly discovered galaxies are listed in Table 3 while those objects which are certainly stars are listed in Table 5 .

On our long-slit spectra we found some further emission-line objects projected near to our targets. These galaxies had good enough signal-to-noise to be fully reduced and they are listed in Table 4 . They were not detected on the Hamburg objective plates because their redshift is higher than $z=0.078$, which is the limit for which the [OIII] $\lambda 5007$ line can be detected due to the cut-off of the IIIa-J emulsion at 5400 (see Paper I for selection effects). These galaxies are designated by the prefix HS followed by the first four digits of the 1950.0 right ascension and declination, plus the suffix $N$ (from neighbours). Their finding charts can be found in Fig. 2, lower panel.

\section{Appendix}

In this appendix we present a few ELGs that were selected from our Region 1 (see Paper I) (the region of the PiscesPerseus Supercluster) and Region 2. These objects were observed after the publication of the first catalogue and therefore were missed from our "List 1".

The observations were done in August 1995 with the Cassegrain Twin Spectrograph at the $3.5 \mathrm{~m}$ telescope at Calar Alto, as a back-up of an observational campaign not dedicated to our project. The details of the observations are given in Table 6 while in Table 7 we list the name of the ELGs, alternative designations (for those objects contained in other catalogues), their coordinates (equinox 1950.0), heliocentric redshifts and emission-lines seen in their spectra. The abbreviations for alternative designations can be found in the List of Abbreviations used for the Table 2. The data reduction follows the same procedure as described in Sect. 2. The errors in the redshifts are around $\Delta z=0.0001-0.0002$. In order to have an external check of our redshift determination we also observed a few candidates that had redshifts in the literature. In Table 8 we give the names of the galaxies observed together with their velocities, both measured and from literature. In the last column we indicate the difference between our values and the reference one. Besides UM27, for which we obtained a big difference, the rest show differences up to 0.0001 in $z$. The finding charts of the newly discovered objects can be found in Fig. 2, upper panel.

Acknowledgements. We would like to thank the Calar Alto staff for their support during the observations. U. Hopp acknowledge the support by the SFB 375 by the Deutsche For-schungsgemeinschaft.

This research has made use of the NASA/IPAC Extragalactic Database (NED) which is operated by the Jet Propulsion Laboratory, California Institute of Technology, under contract with the National Aeronautics and Space Administration.

\section{References}

Hagen H.-J., Groote D., Engels D., Reimers D., 1995, A\&AS 111,195

Hopp U., Kuhn B., Thiele U., Birkle K., Elsässer H., 1995, A\&AS 109, 537

Horne K., 1986, PASP 98, 609

Kojoyan G., Elliott R., Bicay M.D., 1982, ApJS 50, 161

Kuhn B., Hopp U., Elsässer H., 1997, A\&A 318, 405

MacAlpine G.M., Smith S.B., Lewis D.W., 1977, ApJS 34, 95

Popescu C.C., Hopp U., Hagen H., Elsässer H., 1996, A\&AS 116, 43 (Paper I)

Popescu C.C., Hopp U., Elsässer H., 1997, A\&A 328, 756 (Paper II)

Stickel M., Fried J.W., Kúhr H., 1993, A\&AS 98, 393

Terlevich R., Melnick J., Masegosa J., Moles M., Copetti M.V.F., 1991, A\&AS 91, 285 


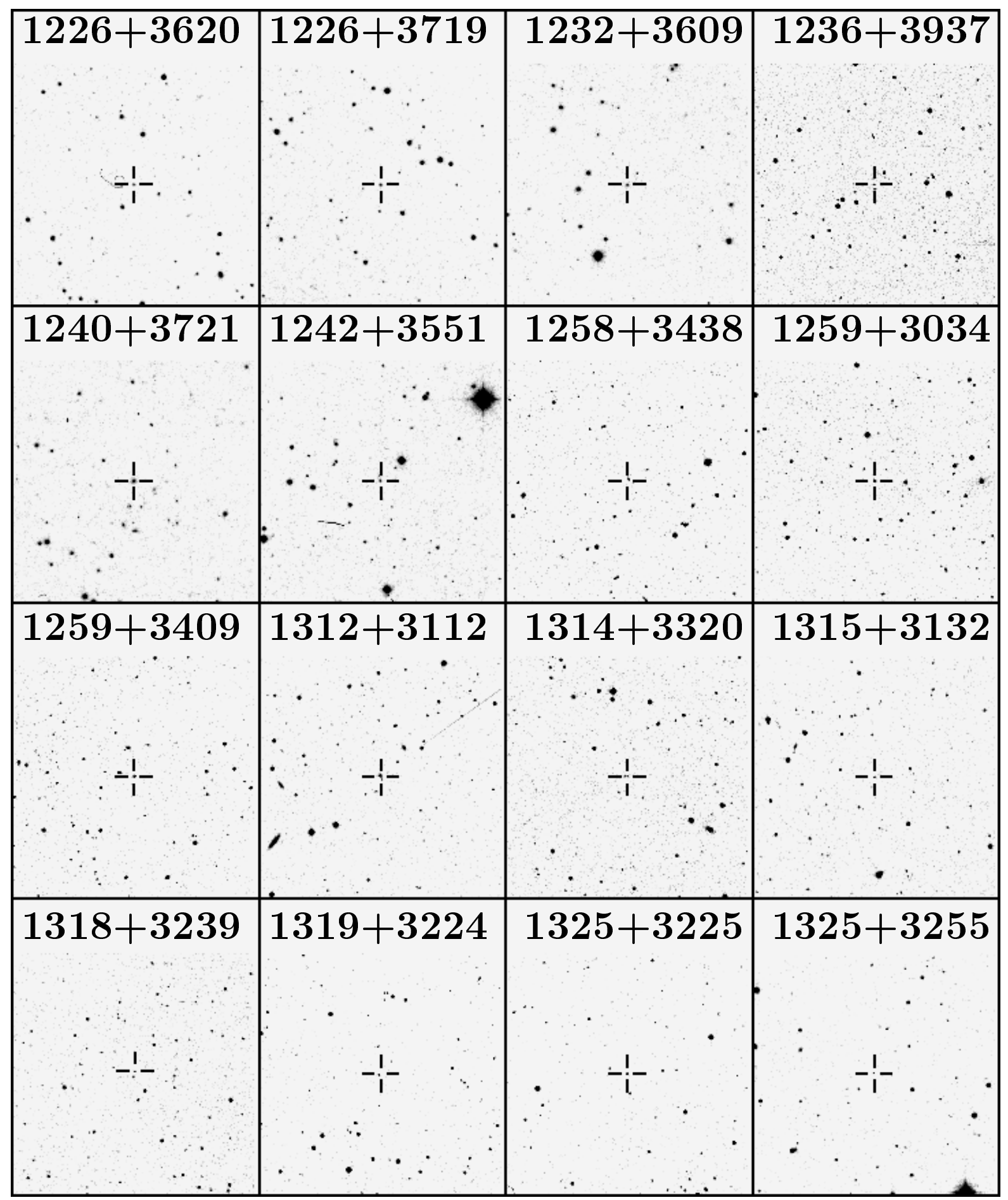

Fig. 1. Finding charts prepared from the Digitised Palomar Sky Survey. Fields are $10^{\prime} \times 10^{\prime}$. North is up, and east is to the left 


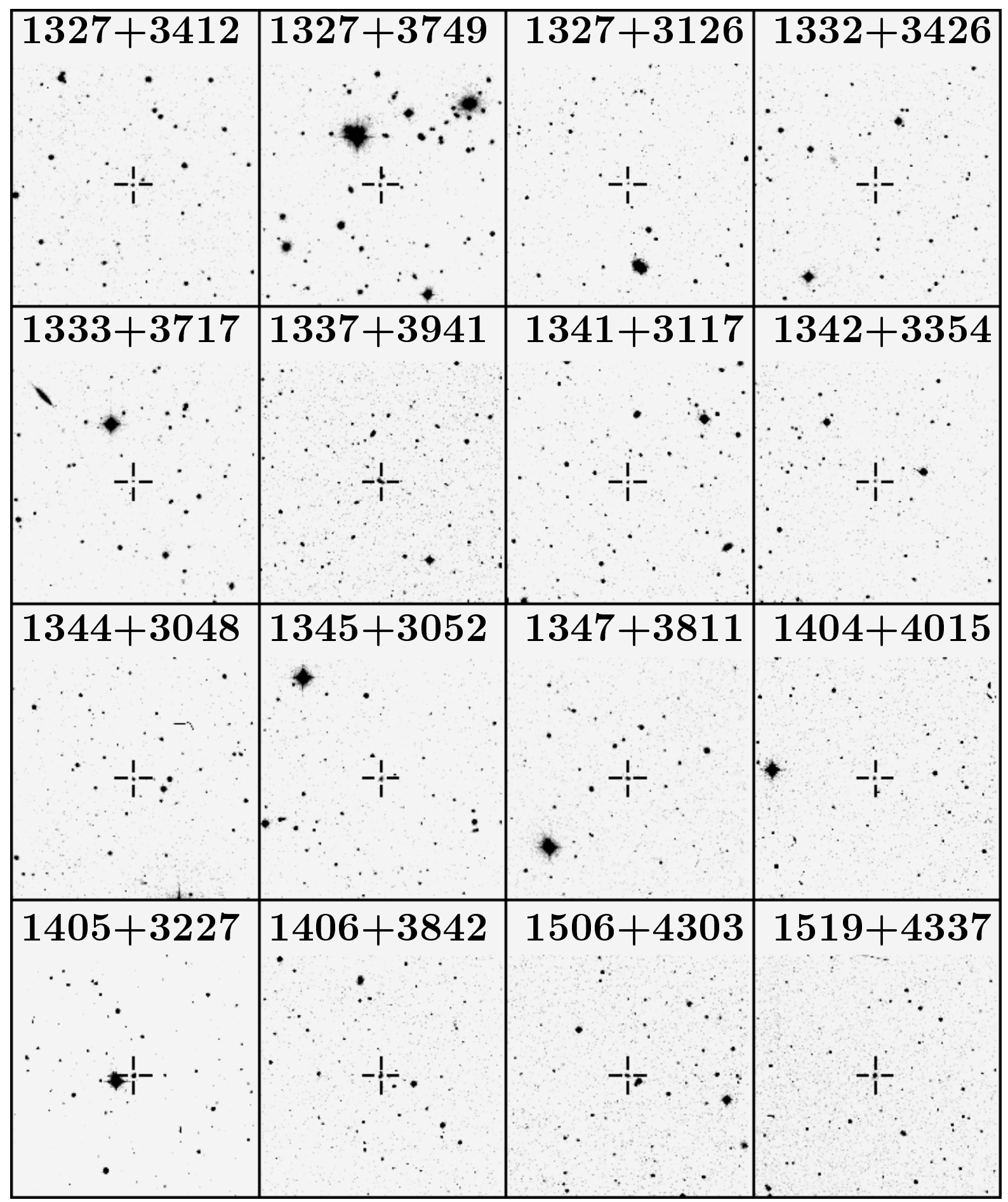

Fig. 1. continued 


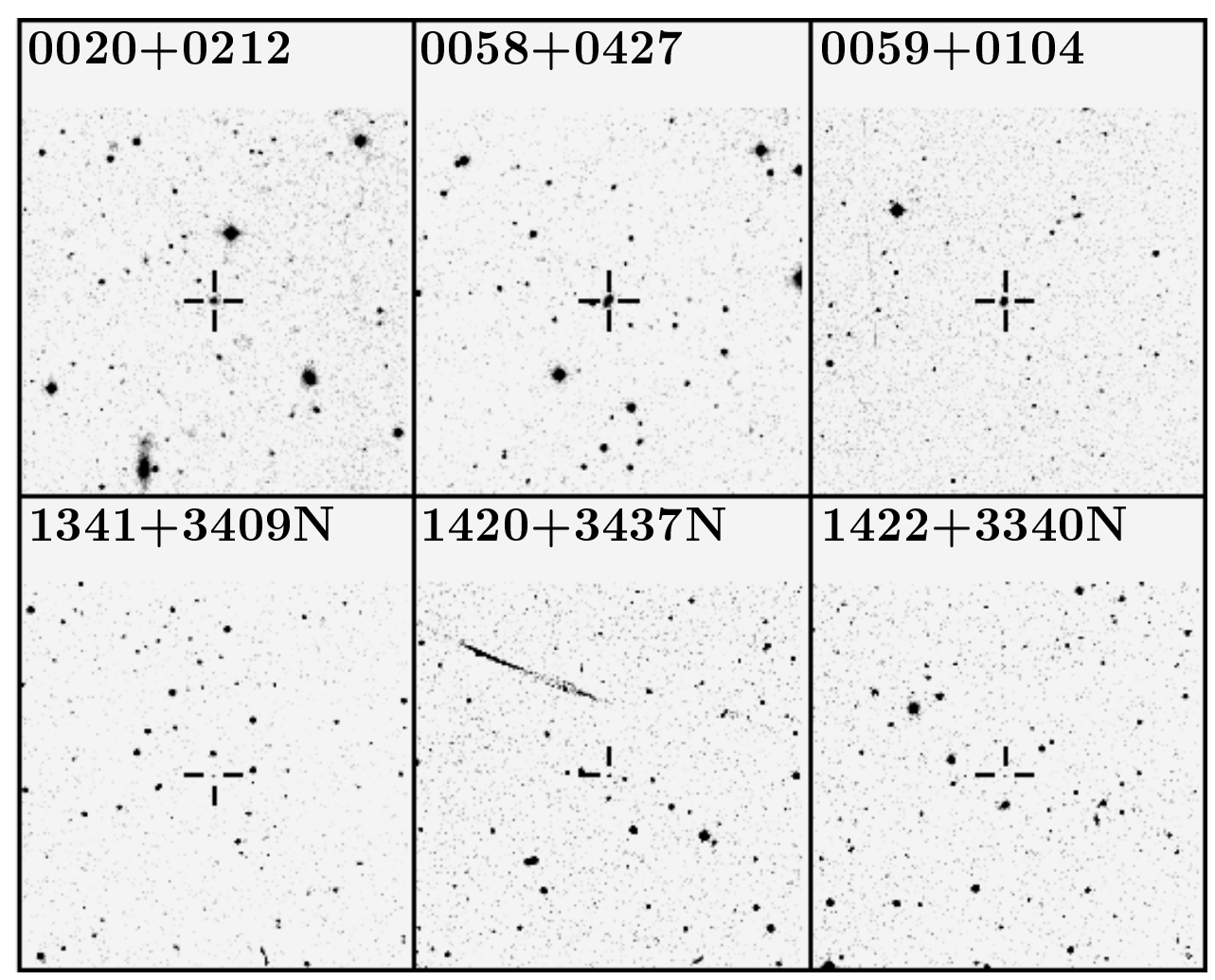

Fig. 2. Finding charts prepared from the Digitised Palomar Sky Survey. Fields are $10^{\prime} \times 10^{\prime}$. North is up, and east is to the left 
Table 2.

\begin{tabular}{|c|c|c|c|c|c|c|}
\hline (1) & $(2)$ & $(3)$ & (4) & (5) & (6) & $(7)$ \\
\hline object name & other names & R.A. & Decl. & redshift & $\mathrm{S}$ & remarks \\
\hline HS1226+3620 & & 122638.6 & +362055 & 0.0680 & $\mathrm{E}$ & \\
\hline HS1232+3609 & & 123209.4 & +360959 & 0.2529 & $\mathrm{E}$ & \\
\hline HS1236+3937 & & 123655.5 & +393733 & 0.0184 & $\mathrm{E}$ & \\
\hline HS1240+3721 & & 124049.0 & +372133 & 0.0218 & $\mathrm{~B}$ & \\
\hline HS1240+3755 & $\begin{array}{l}\text { IRAS } 12409+3755 \\
\text { NPM1G }+37.0370\end{array}$ & 124058.6 & +375523 & 0.0860 & $\mathrm{~B}$ & $\operatorname{IrS}$ \\
\hline & FIRST J124322.5+373858 & & & & & Radio S \\
\hline HS1242+3551 & & 124245.2 & +355134 & 0.1589 & $\mathrm{E}$ & \\
\hline HS1258+3438 & & 125822.2 & +343815 & 0.0248 & $\mathrm{E}$ & \\
\hline HS1259+3034 & & 125915.9 & +303435 & 0.1268 & $\mathrm{~B}$ & abs \\
\hline HS1259+3409 & & 125921.7 & +340933 & 0.1089 & $\mathrm{E}$ & abs \\
\hline HS1310+3801 & PC $1310+3801$ & 131032.3 & +380104 & 0.0230 & $\mathrm{E}$ & \\
\hline HS1312+3112 & & 131218.4 & +311254 & 0.0205 & $\mathrm{E}$ & $*$ \\
\hline $\mathrm{HS} 1314+3320$ & & 131432.0 & +332059 & 0.0515 & $\mathrm{~B}$ & \\
\hline HS1315+3132 & & 131515.9 & +313251 & 0.0315 & $\mathrm{E}$ & \\
\hline HS1318+3239 & & 131844.0 & +323958 & 0.0435 & $\mathrm{E}$ & \\
\hline HS1319+3224 & & 131900.2 & +322407 & 0.0182 & $\mathrm{E}$ & \\
\hline HS1325+3225 & & 132520.1 & +322523 & 0.0504 & $\mathrm{E}$ & \\
\hline HS1325+3255 & & 132559.1 & +325527 & 0.0263 & $\mathrm{E}$ & \\
\hline HS1327+3412 & & 132716.7 & +341203 & 0.2510 & $\mathrm{E}$ & \\
\hline HS1327+3749 & & 132725.6 & +374933 & 0.0543 & $\mathrm{E}$ & \\
\hline $\mathrm{HS} 1327+3126$ & & 132732.0 & +312638 & 0.0568 & $\mathrm{E}$ & \\
\hline HS1327+3135 & UGC 08496 & 132759.3 & +313525 & 0.0161 & $\mathrm{E}$ & G Group \\
\hline
\end{tabular}

VV 069

KUG $1327+315$

CGCG 161 - 071

CGCG 1328.0+3135

$\mathrm{MCG}+05-32-031$

WAS 73

[SMB88] 0742

HS1332+3426

LGG 350:[G93] 005

HS1333+3717

HS1337+3941

HS1341+3117

HS1342+3354

$\mathrm{HS} 1344+3048$

HS1345+3052

HS1347+3811

HS1404+4015

HS1405+3227

IRAS $14057+3227$

IRAS F14057+3227

FIRST J140756.9+321249

HS1406+3842

HS1424+3836

CG 0428

[SP82] 13

HS1440+3120

CG 1252

HS1445+3801

IRAS F14456+3801

CG 0543

\begin{tabular}{|c|c|c|c|c|}
\hline 133227.9 & +342620 & 0.0220 & $\mathrm{E}$ & \\
\hline 133334.6 & +371703 & 0.0569 & $\mathrm{E}$ & \\
\hline $\begin{array}{lll}13 & 37 & 20.7\end{array}$ & +394110 & 0.0025 & $\mathrm{E}$ & \\
\hline 134132.8 & +311751 & 0.0293 & $\mathrm{E}$ & \\
\hline 134203.1 & +335431 & 0.0054 & $\mathrm{E}$ & \\
\hline 134408.9 & +304807 & 0.1694 & B & abs \\
\hline 134527.0 & +305204 & 0.0768 & $\mathrm{E}$ & \\
\hline 134702.4 & +381135 & 0.0103 & $\mathrm{E}$ & \\
\hline 140415.5 & +401504 & 0.1519 & B & \\
\hline 140545.4 & +322701 & 0.0864 & $\mathrm{E}$ & $\begin{array}{l}\text { IrS } \\
\text { abs }\end{array}$ \\
\hline 40638.2 & +384241 & 0.1549 & B & $\begin{array}{l}\text { Radio S } \\
\text { abs }\end{array}$ \\
\hline 42425.8 & +383626 & 0.0218 & $\mathrm{E}$ & \\
\hline 0.2 & +3120 & 0.0525 & $\mathrm{E}$ & \\
\hline 6.1 & +380136 & 0.0353 & $\mathrm{E}$ & IrS \\
\hline
\end{tabular}

[SP82] 23 
Table 2. continued

\begin{tabular}{|c|c|c|c|c|c|c|}
\hline (1) & $(2)$ & (3) & (4) & $(5)$ & (6) & $(7)$ \\
\hline object name & other names & R.A. & Decl. & redshift & $\mathrm{S}$ & remarks \\
\hline HS1506+4303 & & 150642.2 & +430324 & 0.0404 & B & $* *$ \\
\hline HS1509+4409 & & 150956.8 & +440928 & 0.0275 & B & $* *$ \\
\hline HS1519+4337 & & 151940.8 & +433757 & 0.1347 & $\mathrm{E}$ & abs \\
\hline HS1529+4512 & CG 0745 & 152928.7 & +451210 & 0.0231 & $\mathrm{E}$ & \\
\hline
\end{tabular}

Remarks on Table 2:

* The galaxy HS1312+3112 has a galactic star superimposed on the North-Eastern side of its image.

** HS1506+4303 and HS1509+4409 were observed with the Twin Spectrograph, with the same set-up as given in Table 6 (see Appendix).

\section{List of Abbreviations}

abbrev.

reference

$\begin{array}{lll}\text { CG } & \text { Case Galaxy } & 8,11 \\ \text { CGCG } & \text { Catalogue of Galaxies and of Cluster of Galaxies 1968 } & 16 \\ \text { FIRST } & \text { Faint Images of the Radio Sky at Twenty Centimeters } & 1 \\ \text { IRAS } & \text { Infrared Astronomical Satellite Catalogs 1988. The Point Source Catalog } & 3 \\ \text { IRAS F } & \text { Infrared Astronomical Satellite Catalogs 1990. The Faint Source Catalog } & 5 \\ \text { KUG } & \text { Kiso Survey for UV Excess Galaxies } & 12 \\ \text { LGG } & \text { Lyon Group of Galaxies Catalog } & 2 \\ \text { MCG } & \text { Morphological Catalogue of Galaxies } & 14 \\ \text { NPM } & \text { Lick Northern Proper Motion Program } & 4 \\ \text { PC } & \text { Palomar Transit Grism Survey } & 9 \\ \text { [SMB88] } & \text { Slezak, Mars, Bijaoi et al. (1988) } & 10 \\ \text { [SP82] } & \text { Sanduleak \& Pesch (1982) } & 7 . \\ \text { UGC } & \text { Uppsala General Catalogue of Galaxies 1973 } & 6 \\ \text { VV } & \text { Vorontsov-Velyaminov (1959, 1977), Atlas and Catalog of Interacting Galaxies } & 13 \\ \text { WAS } & \text { Wasilewski (1983) } & 15\end{array}$


References for Table 2

1. Becker R.H., White R.L., Helfand D.J., 1995, ApJ 450, 559

2. Garcia A.M., 1993, A\&AS 100, 46

3. Joint IRAS Science Working Group 1988, Infrared Astronomical Satellite Catalogs, 1988, The Point Source Catalog, Version 2.0, NASA RP-1190

4. Klemola A.R., Jones B.F., Hanson R.B., 1987, AJ 94, 501

5. Moshir M., Kopan G., Conrow T., Mccallon H., Hacking P., Gregorich D., Rohrbach G., Melnyk M., Rice W.,

Fullmer L., et al., 1990, Infrared Astronomical Satellite Catalogs, 1990, The Faint Source Catalog, Version 2.0

6. Nilson P., 1973, Uppsala General Catalogue of Galaxies, Nova Acta Regiae Societatis Scientiarum Ser. V, Vol. 1, Uppsala

7. Sanduleak N., Pesch P., 1982, ApJ 258, L11

8. Sanduleak N., Pesch P., 1987, ApJS 63, 809 (Paper IV)

9. Schneider D.P., Schmidt M., Gunn J.E., 1994, AJ 107, 1245

10. Slezak E., Mars G., Bijaoui A., Balkowski C., Fontanelli P., 1988, A\&AS 74, 83

11. Stephenson C.B., Pesch P., MacConnell D.J., 1992, ApJS 82, 471 (Paper XIII)

12. Takase B., Miyauchi-Isobe N., 1987, Ann. Tokio Ast. Obs. 21, 251

13. Vorontsov-Velyaminov B.A., 1959, Atlas and Catalog of Interacting Galaxies, Sternberg. Inst., Moscow State University

14. Vorontsov-Velyaminov B.A., et al., 1962, 1963, 1964, 1968, 1974. Morphological Catalog of Galaxies, Part I-V, Moscow State University

15. Wasilewski A.J., 1983, ApJ 272, 68

16. Zwicky F., Herzog E., Wild P., Karpowicz M., Kowal C., 1961-1968, Catalog of Galaxies and of Clusters of Galaxies, Pasadena: California Institute of Technology 
Table 3. The emission and absorption lines detected in the spectra of the observed objects

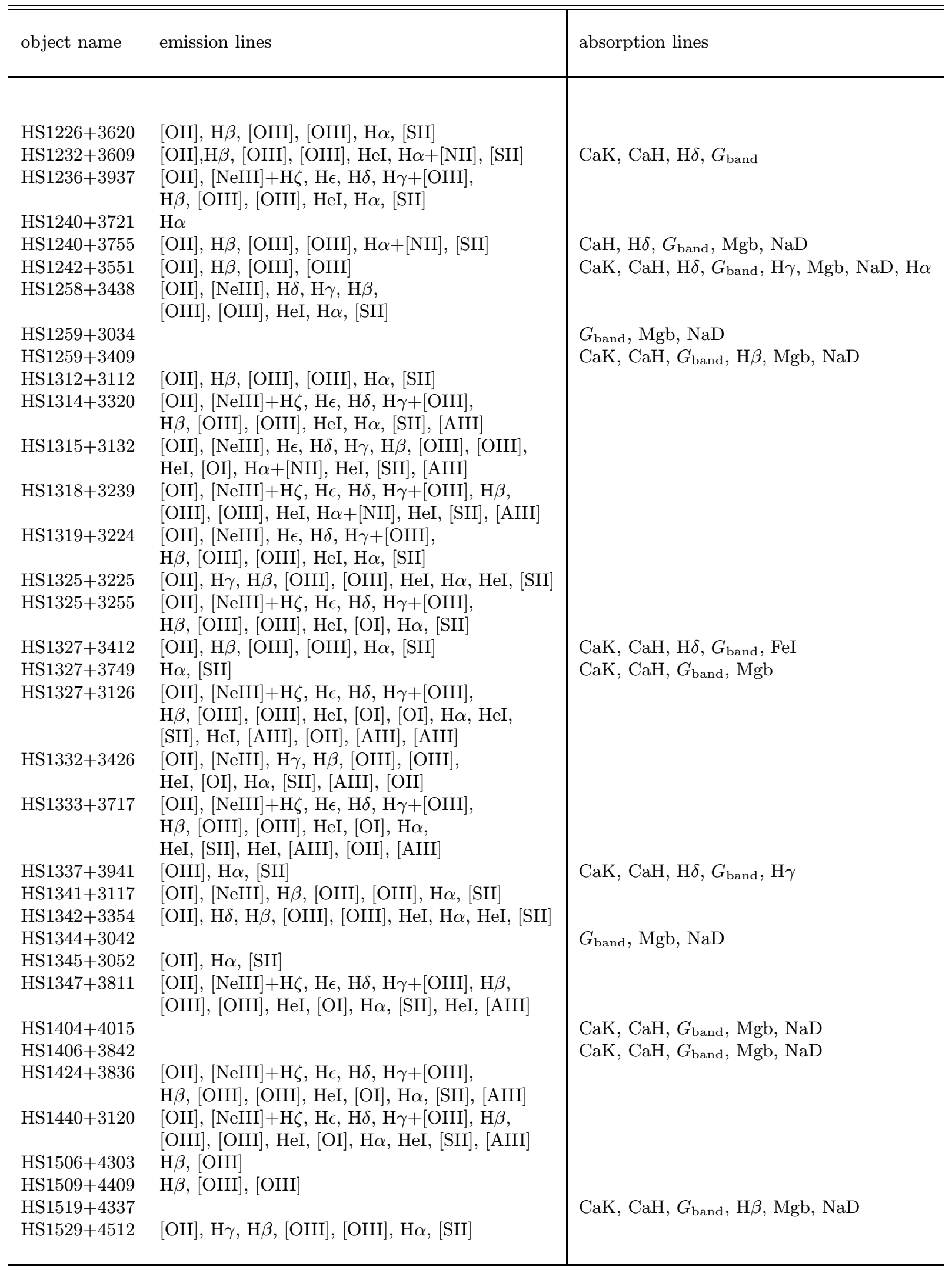


The complete sequence of emission lines listed in Table 3 (wavelengths given in $\AA$ ): [OII] (blend) 3727.45, [NeIII] 3868.76, H $\zeta$ 3889.05, $\mathrm{H} \epsilon$ (blend [NeIII], [OII]) 3970.07 (3967.47, 3967.40), H $\delta$ 4101.6, H $\gamma$ 4340.3, [OIII] 4363.21, H $\beta$ 4861.2, [OIII] 4958.92, [OIII] 5006.85, HeI 5875.99, [OI] (blend with [SII]) 6300.32 (6312.1), [OI] 6363.81, [NII] 6548.2, H $\alpha$ 6562.9, [NII] 6583.6, HeI 6678.1, [SII] (blend) 6723.6, HeI 7065.3, [AIII] 7135.8, [OII] (blend) 7319.9, 7330.2, [AIII] 7751.02, [AIII] 8036.3

Table 4.

\begin{tabular}{|c|c|c|c|c|}
\hline (1) & $(2)$ & (3) & (4) & $(5)$ \\
\hline object name & R.A. & Decl. & redshift & emission lines \\
\hline $\mathrm{HS} 1341+3409 \mathrm{~N}$ & 134150.9 & +340914 & 0.2813 & {$[\mathrm{OII}], \mathrm{H} \alpha$} \\
\hline $\mathrm{HS} 1420+3437 \mathrm{~N}$ & 142059.1 & +343742 & 0.1308 & {$[\mathrm{OII}], \mathrm{H} \beta,[\mathrm{OIII}], \mathrm{H} \alpha$} \\
\hline $\mathrm{HS} 1422+3340 \mathrm{~N}$ & 142253.8 & +334009 & 0.2427 & $\mathrm{H} \alpha$ \\
\hline
\end{tabular}

Table 5. List of identified stars

\begin{tabular}{|c|c|c|c|c|}
\hline object name & coord. & $\mathrm{B}$ & type & abs. lines \\
\hline HS1328+3524 & $132801.5+352434$ & 18.1 & mid-G & $G_{\text {band }}, \mathrm{Mgb}$ \\
\hline HS1344+3640 & $134415.1+364007$ & 19.5 & mid-F & $\mathrm{H} \delta, \mathrm{H} \gamma, \mathrm{H} \beta, \mathrm{Mgb}, \mathrm{H} \alpha$ \\
\hline HS1410+3352 & $141030.5+335246$ & 19.5 & M0-M2 & $\mathrm{NaD}$ \\
\hline $\mathrm{HS} 1513+4028$ & $151353.6+402820$ & 19.5 & late $F$ or early $G$ & $G_{\text {band }}, \mathrm{H} \gamma, \mathrm{H} \beta, \mathrm{Mgb}, \mathrm{NaD}, \mathrm{H} \alpha$ \\
\hline HS1516+4441 & $151619.1+444147$ & 19.3 & $\operatorname{mid} \mathrm{M}$ & \\
\hline HS1524+4255 & $152450.1+425531$ & 19.7 & $A$ or early $F$ & $\mathrm{H} \alpha$ \\
\hline
\end{tabular}

Table 7.

\begin{tabular}{|c|c|c|c|c|}
\hline (1) & $(2)$ & $(3)$ & $(4)$ & $(5)$ \\
\hline object name & other names & R.A. & Decl. & redshift \\
\hline HS0008+0443 & & 000845.9 & +044342 & $0.0389^{*}$ \\
\hline HS0017+0018 & $\begin{array}{l}\mathrm{MCG}+00-02-002 \\
\text { NPM1G }+00.0010\end{array}$ & 001713.5 & +001848 & 0.0177 \\
\hline HS0020+0212 & & 002035.3 & +021258 & 0.0183 \\
\hline HS0053+0606 & $\mathrm{NPM} 1 \mathrm{G}+06.0043$ & 005307.3 & +060639 & 0.0401 \\
\hline HS0058+0427 & & 005833.5 & +042755 & 0.0300 \\
\hline HS0059+0104 & & 005957.6 & +010430 & 0.0164 \\
\hline HS0800+6141 & $\begin{array}{l}\text { UGC } 04196 \\
\text { KUG } 0800+616 \\
\text { MCG }+10-12-069 \\
\text { CGCG } 287-037 \\
\text { CGCG } 0800.0+6142\end{array}$ & 080001.0 & +614121 & 0.0298 \\
\hline HS0838+6253 & & 083845.2 & +625307 & $0.0051^{* *}$ \\
\hline
\end{tabular}


* At the position of HS0008+0443 the NASA/IPAC Extragalactic Database (NED) reports an already known object, namely UM20. This object is assigned with a redshift of $z=0.0830$ (reference: Terlevich et al. 1991), while we determined $z=0.0389$. The galaxy observed by Terlevich et al. (1991) does not have emission lines, while our's has! Trying to understand this discrepancy we looked in the original paper and realised that the authors give other coordinates for UM20, namely $\alpha=00^{\mathrm{h}} 08^{\mathrm{m}} 42.0^{\mathrm{s}}$, $\delta=04^{\circ} 44^{\prime} 00^{\prime \prime}$ (equinox 1950.0). These coordinates are obviously copied from the original UM list of MacAlpine et al. (1977). The coordinates given in NED for UM20 come from the identification of Kojoyan et al. (1982). Probably they do not refer to UM20, but to HS0008+0443. We can only speculate that this misidentification may come from the fact that on the Palomar Sky Survey there seems to be no object at the position indicated in the original list of MacAlpine et al. (1977) or Terlevich et al. (1991). In conclusion we consider that UM20 as observed by Terlevich et al. (1991) is not the same as the object identified by Kojoyan et al. (1982), and also differs from HS0008+0443. It is also obvious that the object observed by Terlevich et al. (1991) must have different coordinates than indicated in their paper.

** The galaxy HS0838+6253 was already observed by us (Paper I), but with a very poor resolution. Here we give an improved redshift determination. 